\title{
Clinical outcome of immunotherapy with dendritic cell vaccine and cytokine-induced killer cell therapy in hepatobiliary and pancreatic cancer
}

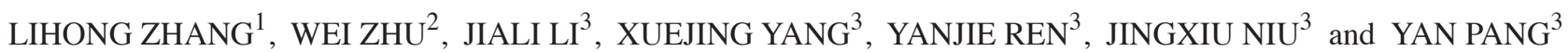 \\ ${ }^{1}$ School of Medicine, NanKai University, Tianjin 300071; ${ }^{2}$ Graduate School of Tianjin Medical University, Tianjin 300070; \\ ${ }^{3}$ Department of Oncology, Tianjin Union Medicine Centre, Tianjin 300121, P.R. China
}

Received January 23, 2015; Accepted October 19, 2015

DOI: $10.3892 / \mathrm{mco} .2015 .660$

\begin{abstract}
The aim of this study was to determine the therapeutic effects of adoptive immunotherapy following dendritic cell (DC) vaccine and cytokine-induced killer (CIK) cell therapy and evaluate its cytotoxicity, survival benefits and quality of life (QOL) changes in patients with hepatobiliary and pancreatic cancer (HPC). We performed a retrospective analysis of 407 clinical cases, including 77 patients with HPC who received immunotherapy with DC vaccine and CIK cells (I group) and 330 patients with similar characteristics who underwent baseline treatment but did not receive immunotherapy [non-immunotherapy (NI) group)] as the control group. After a follow-up period of $294 \pm 207.5$ days, the median survival time (MST) of the two groups was compared using the Kaplan-Meier method. In the I group, $61 \%$ of the patients developed a positive, delayed-type hypersensitivity response and $65 \%$ of the patients exhibited an improvement in QOL. The most notable adverse events included fever $(28 \%)$, insomnia (25\%), anorexia (17\%), skin rash (12\%) and arthralgia $(31 \%)$. No severe toxicities were observed in patients in the I group; in addition, the MST was significantly longer in the I group compared with that in the NI group $(\mathrm{P}=0.014)$. Thus, the DC vaccine and CIK cell therapy was associated with mild adverse effects, but was able to induce an immune response and effectively eliminate tumor cells, thereby improving the QOL and prolonging the MST of the patients.
\end{abstract}

Correspondence to: Professor Yan Pang, Department of Oncology, Tianjin Union Medicine Centre, 190 Jieyuan Road, Tianjin 300121, P.R. China

E-mail: pangyancn@126.com

Abbreviations: CIK, cytokine-induced killer; DC, dendritic cell; DTH, delayed-type hypersensitivity; HPC, hepatobiliary and pancreatic cancer; I, immunotherapy; NI, non-immunotherapy; IL-4, interleukin-4; MST, median survival time; PBMC, peripheral blood mononuclear cell; QOL, quality of life

Key words: pancreatic cancers, dendritic cell, cytokine-induced killer cells, hepatobiliary

\section{Introduction}

Hepatobiliary and pancreatic cancer (HPC) is a major threat to human health, with an increasing incidence over the last few years. Surgery, chemotherapy and radiotherapy are currently the mainstay of treatment for HPC. Although surgery is the first treatment of choice, early-stage cancer is diagnosed in a limited number of patients $(1,2)$. Furthermore, the hepatobiliary and pancreatic systems exhibit a complex anatomical structure and serve important physiological functions. Consequently, HPC symptoms lack specificity and have typically reached an advanced stage at diagnosis. Radical resection of HPC is not typically performed, and the majority of HPCs are associated with a poor prognosis $(3,4)$. Chemotherapy and radiotherapy are the primary treatments for advanced-stage disease; however, these treatments may result in major health issues and are associated with severe treatment-related toxicity. Furthermore, the overall health status of HPC patients is generally poor, which may prevent them from being eligible for these standard therapies $(2,5)$.

In addition to the common factors affecting the prognosis of cancer patients (disease stage, tumor size, lymph node metastasis and radical surgery), individual differences in the immune factors among patients also affect prognosis. As all cancer patients exhibit varying degrees of low immunity and a significant proportion are in an immunosuppressed state, recovering anticancer immunity is a possible approach to cancer treatment. Autologous immune cell therapy has been the fourth most commonly used treatment method for cancer and it is widely used in clinical practice. Immunotherapy stimulates the body's own immune system to fight cancer cells. It has been demonstrated that antigen-specific T-cell dysfunction occurs in several cancer patients (6-9). As a result, tumor cells may escape immune surveillance (10). Restoring proper immune system function may be a viable option for improving cancer treatment and immunotherapy may be a promising and safe approach to cancer eradication (11).

The aim of the present study was to investigate the cellular immune response to autoimmune cell therapy in patients with HPC in terms of delayed-type hypersensitivity (DTH), improvement in the quality of life (QOL), safety and prolonged survival. 


\section{Patients and methods}

Patients. All the patients were treated at the Department of Oncology, Tianjin Union Medicine Center (Tianjin, China). HPC patients who received immunotherapy, including an autologous dendritic cell (DC) vaccine and cytokine-induced killer (CIK) cell therapy comprised the immunotherapy group (I group). All the patients provided informed consent prior to DC-CIK treatment and were required to meet the following inclusion criteria: i) A cytological or histological diagnosis of HPC; ii) hospitalization between January 4, 2012 and December 31, 2012; iii) adequate kidney and liver function and normal coagulation; iv) a Karnofsky performance status score of $>60$; and v) expected survival of $>3$ months.

The control group included patients who satisfied the abovementioned inclusion criteria, but did not receive immunotherapy [non-immunotherapy (NI) group]. The follow-up for all the patients was completed on November 14, 2013. The median survival time (MST) was compared between the two groups and the benefit of the DC vaccine and CIK cell-based immunotherapy regimens was evaluated in terms of DTH and improvement in QOL.

Study design. This retrospective study was an open-label, parallel-group, single-institution, non-randomized study and complied with the class III medical techniques policy of the Ministry of Health of China, as described in the guidelines for treatment with autologous immune cells ( $\mathrm{T}$ cells, natural killer cells) $(12,13)$. The study protocol was approved by the Ethics Committee of the Tianjin Union Medicine Center. Prior to treatment, all the patients were asked to provide written informed consent.

The DC vaccine and CIK cell therapy schedule was as follows: The day on which peripheral blood mononuclear cells (PBMCs) were collected was defined as day 0. On day $8,1 \times 10^{7}$ DCs were infused intravenously once/week for 6 weeks, and on day $11,1 \times 10^{9} \mathrm{CIK}$ cells were infused intravenously once/day for 4 days (Table I).

Preparation of cells. Using the Fresenius Kabi system (Fresenius Kabi AG, Bad Homburg, Germany), PBMCs were collected from each patient under electrocardiographic monitoring. The cells were cultured overnight, then adherent cells (including monocytes) and suspension cells (including lymphocytes) were collected separately (14-16).

Preparation of the DC vaccine. The DC vaccine was prepared according to the following protocol: Isolated PBMCs were resuspended in a serum-free culture medium (Hyclone, Logan, UT, USA). The PBMCs were then seeded into a $175-\mathrm{cm}^{3}$ flask to a concentration of $2-4 \times 10^{6} / \mathrm{ml}$, divided among three bottles and then placed in a $37^{\circ} \mathrm{C}, 5 \% \mathrm{CO}_{2}$ incubator (Thermo Fisher Scientific, Waltham, MA, USA). The cell concentration and the number of bottles used may be adjusted according to the number of cells. After $2 \mathrm{~h}$ of incubation, the $175-\mathrm{cm}^{3}$ flasks were gently agitated; the suspension cells were discarded (or collected and used for culturing CIK cells), then washed 1-2 times with serum-free medium and, following addition of the DC medium, placed again in the $37^{\circ} \mathrm{C}, 5 \% \mathrm{CO}_{2}$ incubator. Autologous serum, granulocyte-macrophage
Table I. Regimen of dendritic cells (DCs) + cytokine-induced killer (CIK) cell therapy.

\begin{tabular}{cll}
\hline Day & \multicolumn{1}{c}{ Therapy } & \multicolumn{1}{c}{ Functions } \\
\hline 0 & Collection of leukocyte fraction & \\
8 & Reinfusion of DC1 i.v. & Vaccination 1 \\
11 & Reinfusion of CIK1 i.v. & Against CC \\
12 & Reinfusion of CIK2+CIK3 i.v. & Against CC \\
13 & Reinfusion of CIK4 i.v. & Against CC \\
14 & Reinfusion of CIK4+ CIK5 i.v. & Against CC \\
15 & Reinfusion of DC2 i.v. & Vaccination 2 \\
22 & Reinfusion of DC3 i.v. & Vaccination 3 \\
29 & Reinfusion of DC4 i.v. & Vaccination 4 \\
36 & Reinfusion of DC5 i.v. & Vaccination 5 \\
43 & Reinfusion of DC6 i.d. & Vaccination 6 \\
\hline
\end{tabular}

i.v., intravenous; i.d., intradermal; CC, cancer cell.

colony-stimulating factor, interleukin (IL)-4 and tumor necrosis factors (all from PeproTech EC, Ltd., London, UK) were added on the 3rd day. The tumor antigen (Peprotech, Inc., Rocky Hill, NJ, USA) was added on the 5th day and the ripening factor was added following addition of the tumor antigen. Flat blood tests were performed and the flasks were again placed in the incubator for $2 \mathrm{~h}$. After the flasks were washed twice with serum-free 1640 medium, the DC culture solution was added and the flasks were placed back in the incubator. DC cells were collected between the 7th and 8th days and then washed three times with saline. The quality of the preparation was determined at the same time.

Preparation of CIK cells. The CIK cells were prepared according to the following protocol: The culture bottles were pre-coated with a $5 \mu \mathrm{g} / \mathrm{ml}$ final concentration of CIK reagents before preparing the CIK cells, then placed in a $4^{\circ} \mathrm{C}$ environment overnight and washed with phosphate-buffered saline (PeproTech EC, Ltd.) 2-3 times on day 0. The CIK cells were resuspended in the initial CIK cell medium at a concentration of $2-3 \times 10^{6} / \mathrm{ml}$ and spread into a culture bag according to the number of cells. Autologous plasma was then added and cultured in a $37^{\circ} \mathrm{C}, 5 \% \mathrm{CO}_{2}$ incubator (PeproTech EC, Ltd.). IL-2, interferon $2 \gamma$ (both from PeproTech EC, Ltd.) and CD3 monoclonal antibody (100 $\mu \mathrm{g} / \mathrm{ul}$; cat. no 20150605; Beckman Coulter, Inc., Brea, CA, USA) were added using 5-ml disposable syringes with needles. To ensure that all factors were added in the culture bag, the aspiration was repeated five times, after which the culture bags were placed in the $37^{\circ} \mathrm{C}, 5 \% \mathrm{CO}_{2}$ incubator. Samples were prepared and counted every 2-3 days using 2-ml disposable syringes with needles. Appropriate additional CIK expansion bottle medium was added according to the results of the count in order to maintain a CIK cell concentration of $1-3 \times 10^{6} / \mathrm{ml}$ and the total number of cells was recorded at the same time.

Cell quality control. The immune phenotype markers of HLA2DR, CD86 and CD83 were used for DCs; CD3, CD8 and CD56 were used for CIK cells. The bacteria, endotoxin 
Table II. Immune phenotype markers.

\begin{tabular}{lccc}
\hline Cell types & Early separation & Reagents & Cell surface phenotype \\
\hline DCs & Adherent cells (monocytes) & GM-CSF, IL-4, TNF, Ag & $\mathrm{CD}^{\mathrm{a}} 3^{+}, \mathrm{CD}^{+}, \mathrm{HLA}^{+} \mathrm{DR}^{+}$ \\
CIK cells & Suspension cells (lymphocytes) & IFN2, CD3McAb, IL-2 & $\mathrm{CD}^{+}, \mathrm{CD}^{+}, \mathrm{CD}^{+} 6^{+}$
\end{tabular}

${ }^{\text {aDCs }}$ were presented with different tumor antigens that stimulate the immunoreactivity of DCs and promote cell proliferation, thereby enhancing the killing effect. DCs, dendritic cells; CIK, cytokine-induced killer; GM-CSF, granulocyte-macrophage colony-stimulating factor; IL, interleukin; TNF, tumor necrosis factor; Ag, antigen; IFN, interferon; McAb, monoclonal antibody.

Table III. DTH, QOL and safety (data loss, $n=5$ ).

\begin{tabular}{lcc}
\hline Results & Degree & No. $(\%)$ \\
\hline DTH & $>10 \mathrm{~mm}$ & $12(16.5)$ \\
& $5-10 \mathrm{~mm}$ & $12(16.5)$ \\
& $2-5 \mathrm{~mm}$ & $18(25.0)$ \\
& $<2 \mathrm{~mm}$ & $30(42.0)$ \\
QOL & 4 & $9(13.0)$ \\
& 3 & $13(18.0)$ \\
& 2 & $13(18.0)$ \\
Fever & 1 & $12(16.0)$ \\
& 0 & $25(35.0)$ \\
& $>39^{\circ} \mathrm{C}$ & $9(13.0)$ \\
& $38-39^{\circ} \mathrm{C}$ & $11(15.0)$ \\
Skin rash & $<38^{\circ} \mathrm{C}$ & $10(14.0)$ \\
& Cold symptoms & $5(7.0)$ \\
Ansomnia & None & $37(51.0)$ \\
& Local injection & $8(11.0)$ \\
Arthralgia & Systemic & $1(1.0)$ \\
& None & $63(88.0)$ \\
& - & $22(31.0)$ \\
& - & $12(17.0)$ \\
& - & $18(25.0)$ \\
& &
\end{tabular}

DTH, delayed-type hypersensitivity; QOL, quality of life.

and fungus levels of the cultured samples satisfied the release criteria for infusion (Table II).

DTH testing, QOL and safety. To determine DTH, $4 \mu \mathrm{g}$ of tumor lysate was injected intradermally 1 week after the last infusion and the results were read $48 \mathrm{~h}$ later. The diameter of the induration corresponded to the result, i.e., $>10 \mathrm{~mm}$ was considered as strongly positive, $5-10 \mathrm{~mm}$ as positive, $2-5 \mathrm{~mm}$ as weakly positive and $<2 \mathrm{~mm}$ as negative. Fever, insomnia, anorexia, arthralgia and skin rash were evaluated as adverse effects during therapy. Certain patients developed $\geq 2$ events. The general status in terms of physical strength, appetite, sleep and body weight were evaluated as QOL indices. QOL scores were defined as follows: 0 , none of the 4 indices; 1 , any one of the 4 indices; 2 , any 2 of the 4 indices; 3 , any 3 of the 4 indices; and 4, all indices. The degrees of 4, 3, 2 and 1 were considered as an improvement in the general status (Table III) $(15,16)$.
Table IV. Comparison of median survival time (MST) between the immunotherapy (I) and the non-immunotherapy (NI) control groups.

\begin{tabular}{lccccc}
\hline Groups & No. & MST, days & $\Delta$ MST, days & $\chi^{2}$ & P-value \\
\hline I & 31 & 134 & 126 & 37.121 & 0.014 \\
NI & 101 & 115 & & & \\
\hline
\end{tabular}

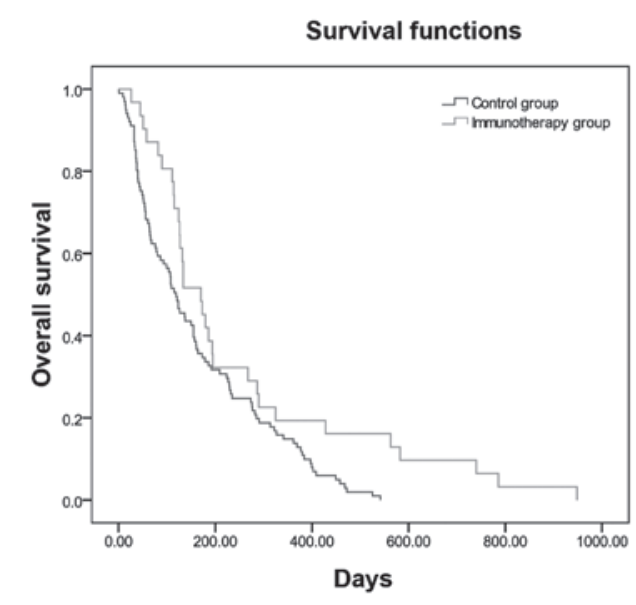

Figure 1. Median survival time curves by the Kaplan-Meier estimate in the immunotherapy and non-immunotherapy (control) groups $(\mathrm{P}=0.014)$.

Overall survival. Overall survival was measured from the time of enrollment to the date of death and compared between groups using the Kaplan-Meier method (Table IV, Fig. 1).

Data collection and statistical analysis. Clinical data were collected from the Inpatients Electronic Medical Records of Tianjin Union Medicine Center, re-analyzed and documented for use with the EpiData software, version 3.02 (Epidata Association, Odense, Denmark). Statistical analyses were performed using the SAS statistical software package (SAS Institute Inc., Cary, NC, USA). The MST curves were calculated using the Kaplan-Meier method. The associations between variables were compared using the Pearson's Chi-square test. $\mathrm{P}<0.05$ was considered to indicate a statistically significant difference.

\section{Results}

Patient characteristics. A total of 407 patients (270 men and 137 women) were enrolled in this study, with a median 
Table V. Patient characteristics.

\begin{tabular}{|c|c|c|c|}
\hline \multirow[b]{2}{*}{ Characteristics } & \multirow[b]{2}{*}{$\begin{array}{c}\text { Total } \\
(\mathrm{n}=407)\end{array}$} & \multicolumn{2}{|c|}{ Groups, no. (\%) } \\
\hline & & $\begin{array}{c}\mathrm{I} \\
77(19.0)\end{array}$ & $\begin{array}{c}\text { NI } \\
330(81.0)\end{array}$ \\
\hline \multicolumn{4}{|l|}{ Age, years } \\
\hline Range & $37-93$ & $37-86$ & $40-93$ \\
\hline Mean $\pm \mathrm{SD}$ & $66.4 \pm 11.5$ & $61.6 \pm 12.2$ & $67.5 \pm 11.1$ \\
\hline \multicolumn{4}{|l|}{ Gender } \\
\hline Male & 270 & $52(19.0)$ & $218(81.0)$ \\
\hline Female & 137 & $25(18.0)$ & $112(82.0)$ \\
\hline \multicolumn{4}{|l|}{ Tumor location } \\
\hline Hepatocellular $\mathrm{Ca}$ & 157 & $36(23.0)$ & $121(77.0)$ \\
\hline Gallbladder $\mathrm{Ca}$ & 46 & $6(12.0)$ & $40(88.0)$ \\
\hline CholangioCa & 54 & $6(11.0)$ & $48(89.0)$ \\
\hline Pancreatic $\mathrm{Ca}$ & 150 & $29(19.0)$ & $121(81.0)$ \\
\hline \multicolumn{4}{|l|}{$\begin{array}{l}\text { Treatment baseline } \\
\text { immunotherapy }\end{array}$} \\
\hline Surgery & 124 & $10(8.0)$ & $114(92.0)$ \\
\hline Radiotherapy & 33 & $15(45.0)$ & $18(55.0)$ \\
\hline Chemotherapy & 44 & $21(48.0)$ & $23(52.0)$ \\
\hline $\begin{array}{l}\text { Chinese herbal } \\
\text { treatment }\end{array}$ & 36 & $23(64.0)$ & $13(36.0)$ \\
\hline DC-CIK & 77 & $77(100.0)$ & $0(0.0)$ \\
\hline
\end{tabular}

I, immunotherapy; NI, non-immunotherapy; SD, standard deviation; $\mathrm{Ca}$, carcinoma; DC, dendritic cell; CIK, cytokine-induced killer.

age of $66.4 \pm 11.5$ years (range, 37-93 years). Among these, 157 patients had hepatocellular cancer, 46 had gallbladder cancer, 54 had cholangiocarcinoma and 150 had pancreatic cancer. Of the 407 patients, 124 underwent primary tumor resection, 33 received radiotherapy, 44 received chemotherapy and 36 received Chinese medicine treatment. All these treatments were administered within the 3 months preceding the initiation of immunotherapy (Table V). The characteristics of the patients in the two groups were well-balanced with respect to the evaluated factors, including age, gender, diagnostic methods and tumor type. The treatment strategies also did not differ significantly between the two groups.

DTH, QOL and adverse effects. A DTH skin test used to assess the immune response to cell therapy for all patients and the results were recorded for 72 of the 77 patients in the I group. Twelve patients $(16.5 \%)$ had a strongly positive response; $12(16.5 \%)$ had a weakly positive response; $18(25 \%)$ had a positive result; and $30(42 \%)$ patients failed to show an immune response. Thus, $42(58 \%)$ patients in total exhibited a positive effect. QOL was recorded for 72 of the 77 patients in the I group. Of these, $9(13 \%)$ patients exhibited improvements in all QOL events; 13 (18\%) exhibited improvements in 3 events; another 13 (18\%) exhibited improvements in 2 events; $12(16 \%)$ exhibited improvement in 1 event; and $25(35 \%)$ exhibited no improvements of the QOL factors. Adverse effects were assessed in 72 of the 77 patients in the I group. Of these,
$37(51 \%)$ did not have fever or cold symptoms and $5(7 \%)$ developed cold symptoms. A total of 30 patients developed fever: Low fever occurred in $10(14 \%)$, moderate fever in $11(15 \%)$ and high fever in 9 patients (13\%). A total of 12 patients $(17 \%)$ developed anorexia; 18 (25\%) developed insomnia; 22 (31\%) developed arthralgia; and $8(11 \%)$ developed skin rash (located at the intradermal injection site in 7 cases and on the upper as well as the lower body in 1 case; Table III).

Comparison of the MST between the I and NI groups. The MST differed between the I and NI groups (134 vs. 115 days, respectively; Table IV) and it was significantly longer (19 days) in the I group compared with that in the NI group $(\mathrm{P}=0.014)$. The overall survival curves for the I and NI groups are shown in Fig. 1.

\section{Discussion}

Biological treatment will likely become a major part of standard cancer therapy and autologous cell immunotherapy is one of the most important tumor biological treatments. DCs were first identified in 1973 by Steinman and Cohn, revealing the enormous potential of these cells in immunotherapy (17). Steinman was awarded the 2011 Nobel Prize in medicine and physiology, focusing attention once again on DCs. DCs may prime a primary as well as a secondary immune response against cancer and function as antigen-presenting cells (18). DCs are currently considered the most powerful antigen-presenting cells in the body; they effectively present tumor antigens to $\mathrm{T}$ lymphocytes and function as a bridge between the tumor cells and T cells (7,19). CIK cells exhibit strong antitumor activity and non-major histocompatibility complex-restricted tumor-killing characteristics $(10,20)$. A number of studies suggest that CIK cells co-cultured with DCs exhibit stronger tumoricidal effects and have already produced better results in different tumor types $(21,22)$. CIK cells have cytotoxic properties and are able to kill tumor cells directly as well as indirectly, through stimulation of the host immune system. CIK cells thus have the potential to eradicate residual cancer cells, thereby preventing recurrence following tumor resection. These properties have led to CIK cells being included in immunotherapy strategies against cancer (1,23-25). Immunotherapy is commonly used together with surgery, chemotherapy, radiotherapy, traditional Chinese medicine and other cancer therapies (26-29). Immune cell therapy may alleviate the adverse effects of chemotherapy and surgery to promote the recovery of the patient's immunity. Several cancer patients with advanced-stage disease are unable to tolerate surgery and radiotherapy, whereas multicycle chemotherapy may also cause severe bone marrow suppression, drug resistance and other adverse effects. Thus, these patients are not suitable for further chemotherapy and are only able to receive supportive treatment. Therefore, there is an urgent need to improve the QOL of these patients and enable them to continue to receive effective therapy $(30,31)$. Adoptive cell immunotherapy provides a novel approach to solving this problem. In our study, the MST was significantly different between the I and NI groups. Combining immunotherapy with surgery, radiotherapy and chemotherapy may exert a synergistic effect on survival compared with the each method alone. Immunotherapy is a new promising approach to cancer therapy. 
DC-CIK adoptive immunotherapy bears great potential and its antitumor efficacy has repeatedly been demonstrated in clinical practice. With the rapid advances in molecular biology, immunology and other biological disciplines, the therapeutic potential of DC-CIK is expected to be further developed.

In conclusion, our findings demonstrated that autoimmune cell therapy is a viable treatment option for HPC patients. The QOL and overall survival of these patients were improved, without severe adverse effects $(19,23,32)$. The mortality rate was lower in the I group compared with that in the NI group and the overall survival was prolonged, proving that immunotherapy is an effective method for cancer treatment $(10,33)$.

\section{Acknowledgements}

The present study was partially supported by the Tianjin Municipal Science and Technology Commission (grant no. 12ZCDZSY17100). The authors would like to thank Shanghai Claison Biotechnology Co. Ltd, Shanghai, China, for providing the method of preparation of the immunocytes.

\section{References}

1. Tan G, Zhang X, Feng H, Luo H and Wang Z: The therapeutic effect of cytokine-induced killer cells on pancreatic cancer enhanced by dendritic cells pulsed with K-ras mutant peptide. Clin Dev Immunol 2011: 649359, 2011.

2. Wong $\mathrm{HH}$ and Lemoine NR: Biological approaches to therapy of pancreatic cancer. Pancreatology 8: 431-461, 2008.

3. Ghanaati H, Alavian SM, Jafarian A, et al: Imaging and imaging-guided interventions in the diagnosis and management of hepatocellular carcinoma (HCC) - review of evidence. Iran J Radiol 9: 167-177, 2012

4. Dhanasekaran R, Limaye A and Cabrera R: Hepatocellular carcinoma: Current trends in worldwide epidemiology, risk factors, diagnosis and therapeutics. Hepat Med 4: 19-37, 2012.

5. Pardee AD and Butterfield LH: Immunotherapy of hepatocellular carcinoma: Unique challenges and clinical opportunities. Oncoimmunology 1: 48-55, 2012.

6. Wang HY and Wang RF: Enhancing cancer immunotherapy by intracellular delivery of cell-penetrating peptides and stimulation of pattern-recognition receptor signaling. Adv Immunol 114: 151-176, 2012.

7. Nowak M and Schmidt-Wolf IG: Natural killer T cells subsets in cancer, functional defects in prostate cancer and implications for immunotherapy. Cancers (Basel) 3: 3661-3675, 2011.

8. Liu Y, Butterfield LH, Fu X, Song Z, Zhang X, Lu C, Ding G and $\mathrm{Wu} \mathrm{M}$ : Lentivirally engineered dendritic cells activate AFP-specific T cells which inhibit hepatocellular carcinoma growth in vitro and in vivo. Int J Oncol 39: 245-253, 2011

9. Condotta SA, Cabrera-Perez J, Badovinac VP and Griffith TS: T-cell-mediated immunity and the role of TRAIL in sepsis-induced immunosuppression. Crit Rev Immunol 33 $23-40,2013$

10. Wang S, Zhang H, Liu C, Jiao X, Liu D, Du W, He Y, Zhang Z, Wu X, Wang J, et al: Human leukocyte antigen-haploidentical donor-derived cytokine-induced killer cells are safe and prolong the survival of patients with advanced non-small cell lung cancer. Oncol Lett 8: 2727-2733, 2014

11. Wang M, Shi SB, Qi JL, Tang XY and Tian J: S-1 plus CIK as second-line treatment for advanced pancreatic cancer. Med Oncol 30: 747, 2013

12. Charoentong P, Angelova M, Efremova M, Gallasch R, Hackl H, Galon J and Trajanoski Z: Bioinformatics for cancer immunology and immunotherapy. Cancer Immunol Immunother 61 : 1885-1903, 2012

13. Sabado RL and Bhardwaj N: Directing dendritic cell immunotherapy towards successful cancer treatment. Immunotherapy 2 : 37-56, 2010.

14. Cui Y, Yang X, Zhu W, Li J, Wu X and Pang Y: Immune response, clinical outcome and safety of dendritic cell vaccine in combination with cytokine-induced killer cell therapy in cancer patients. Oncol Lett 6: 537-541, 2013.
15. Zhu H, Yang X, Li J, Ren Y, Zhang T, Zhang C, Zhang J, Li J and Pang Y: Immune response, safety and survival and quality of life outcomes for advanced colorectal cancer patients treated with dendritic cell vaccine and cytokine-induced killer cell therapy. Biomed Res Int 2014: 603871, 2014.

16. Niu J, Ren Y, Zhang T, Yang X, Zhu W, Zhu H, Li J, Li J and Pang Y: Retrospective comparative study of the effects of dendritic cell vaccine and cytokine-induced killer cell immunotherapy with that of chemotherapy alone and in combination for colorectal cancer. Biomed Res Int 2014: 214727, 2014.

17. Nussenzweig MC, Steinman RM, Gutchinov B and Cohn ZA: Dendritic cells are accessory cells for the development of anti-trinitrophenyl cytotoxic T lymphocytes. J Exp Med 152: 1070-1084, 1980

18. Salman B, Zhou D, Jaffee EM, Edil BH and Zheng L: Vaccine therapy for pancreatic cancer. Oncoimmunology 2: e26662, 2013.

19. Tada F, Abe M, Hirooka M, Ikeda Y, Hiasa Y, Lee Y, Jung NC, Lee WB, Lee HS, Bae YS, et al: Phase I/II study of immunotherapy using tumor antigen-pulsed dendritic cells in patients with hepatocellular carcinoma. Int J Oncol 41: 1601-1609, 2012.

20. Ma Y, Xu YC, Tang L, Zhang Z, Wang J and Wang HX Cytokine-induced killer (CIK) cell therapy for patients with hepatocellular carcinoma: Efficacy and safety. Exp Hematol Oncol 1: 11, 2012.

21. Martin CA, Kurkowski DL, Valentino AM and Santiago-Schwarz F: Increased intracellular, cell surface and secreted inducible heat shock protein 70 responses are triggered during the monocyte to dendritic cell (DC) transition by cytokines independently of heat stress and infection and may positively regulate DC growth. J Immunol 183: 388-399, 2009.

22. Liu C, Suksanpaisan L, Chen YW, Russell SJ and Peng KW: Enhancing cytokine-induced killer cell therapy of multiple myeloma. Exp Hematol 41: 508-517, 2013.

23. Schmeel FC, Schmeel LC, Gast SM and Schmidt-Wolf IG: Adoptive immunotherapy strategies with cytokine-induced killer (CIK) cells in the treatment of hematological malignancies. Int J Mol Sci 15: 14632-14648, 2014.

24. Rettinger E, Glatthaar A, Abhari BA, et al: SMAC mimetic BV6 enables sensitization of resistant tumor cells but also affects cytokine-induced killer (CIK) cells: A potential challenge for combination therapy. Front Pediatr 2: 75, 2014.

25. Jiang J, Wu C and Lu B: Cytokine-induced killer cells promote antitumor immunity. J Transl Med 11: 83, 2013.

26. Liu H, Song J, Yang Z and Zhang X: Effects of cytokine-induced killer cell treatment combined with FOLFOX4 on the recurrence and survival rates for gastric cancer following surgery. Exp Ther Med 6: 953-956, 2013.

27. Zhong R, Han B and Zhong H: A prospective study of the efficacy of a combination of autologous dendritic cells, cytokine-induced killer cells and chemotherapy in advanced non-small cell lung cancer patients. Tumour Biol 35: 987-994, 2014.

28. Yuanying Y, Lizhi N, Feng M, Xiaohua W, Jianying Z, Fei Y, Feng J, Lihua H, Jibing C, Jialiang L, et al: Therapeutic outcomes of combining cryotherapy, chemotherapy and DC-CIK immunotherapy in the treatment of metastatic non-small cell lung cancer. Cryobiology 67: 235-240, 2013.

29. Wang YY, Wang YS, Liu T, Yang K, Yang GQ, Liu HC, Wang SS and Yang JL: Efficacy study of CyberKnife stereotactic radiosurgery combined with CIK cell immunotherapy for advanced refractory lung cancer. Exp Ther Med 5: 453-456, 2013

30. Niu LZ, Li JL, Zeng JY, Mu F, Liao MT, Yao F, Li L, Liu CY, Chen JB,Zuo JS, et al: Combination treatment with comprehensive cryoablation and immunotherapy in metastatic hepatocellular cancer. World J Gastroenterol 19: 3473-3480, 2013

31. Li W, Xu LP, DI Zhao L, Wang L, Zhang Y, Gao QL and Mai L: Cytokine-induced killer cell therapy for advanced pancreatic adenocarcinoma: A case report and review of the literature. Oncol Lett 5: 1427-1429, 2013.

32. Nakamoto Y, Mizukoshi E, Tsuji H, Sakai Y, Kitahara M, Arai K, Yamashita T, Yokoyama K, Mukaida N, Matsushima K, et al: Combined therapy of transcatheter hepatic arterial embolization with intratumoral dendritic cell infusion for hepatocellular carcinoma: Clinical safety. Clin Exp Immunol 147: 296-305, 2007.

33. Jiang JT, Shen YP, Wu CP, Zhu YB, Wei WX, Chen LJ, Zheng X, Sun J, Lu BF and Zhang XG: Increasing the frequency of CIK cells adoptive immunotherapy may decrease risk of death in gastric cancer patients. World J Gastroenterol 16: 6155-6162, 2010. 